\title{
TNF-a-induced LRG1 promotes angiogenesis and mesenchymal stem cell migration in the subchondral bone during osteoarthritis
}

\author{
Yiyun Wang ${ }^{1}$, Jiajia Xu ${ }^{1}$, Xudong Zhang ${ }^{1}$, Chuandong Wang ${ }^{1}$, Yan Huang ${ }^{1}$, Kerong Dai ${ }^{\star, 1,2}$ and Xiaoling Zhang ${ }^{\star, 1,2}$
}

The incomplete understanding of aberrant neovascularization, which contributes to osteoarthritis suggests that additional modulators have yet to be identified. Our objective was to identify the role of Leucine-rich-alpha-2-glycoprotein1 (LRG1), a new regulator of pathogenic angiogenesis, in osteoarthritis progression and to develop effective treatment strategies. In this study, immunohistochemistry showed that LRG1 was increased in the subchondral bone and articular cartilage in anterior cruciate ligament transection (ACLT) mice. Further studies were focused on the role of LRG1 in osteoarthritis. Results showed that LRG1 promoted angiogenesis and mesenchymal stem cells (MSC) migration, which contribute to aberrant bone formation in the subchondral bone. Moreover, tumor necrosis factor- $\alpha$ (TNF- $\alpha$ ), not interleukin-1 $\beta$ (IL-1 $\beta$ ), IL-6 or IL-17, induced the LRG1 expression in human umbilical vein endothelial cells and this effect was inhibited by p38 mitogen-activated protein kinase or NF- $\kappa$ B inhibitor. Notably, inhibition of TNF- $\alpha$ and LRG1 activity by Lenalidomide, an inhibitor of TNF- $\alpha$ production, in ACLT mice attenuated degeneration of osteoarthritis articular cartilage. This study shows that TNF- $\alpha$ is the predominant proinflammatory cytokine that induces the secretion of LRG1. LRG1 contributes to angiogenesis-coupled de novo bone formation by increasing angiogenesis and recruiting MSCs in the subchondral bone of osteoarthritis joints. Inhibition of TNF- $\alpha$ and LRG1 by Lenalidomide could be a potential therapeutic approach.

Cell Death and Disease (2017) 8, e2715; doi:10.1038/cddis.2017.129; published online 30 March 2017

Osteoarthritis (OA), a degenerative joint disorder, is the leading cause of pain and disability in the aging population. OA has been projected to affect 67 million people by 2030 in the United States, resulting in adverse effects on individuals and health system burden. ${ }^{1}$ Unfortunately, although many defined factors (such as biomechanical, metabolic, inflammatory or genetic factors) are associated with $\mathrm{OA}$, the exact pathogenesis remains unclear. $^{2}$ Therefore, the lack of disease-modifying treatment necessitates joint replacement for some severely affected patients. ${ }^{3}$

Angiogenesis contributes to structural damage and pain in OA progression. ${ }^{4,5}$ Healthy articular cartilage is without vessels and nerves, but during OA progression, cells within the subchondral bone express angiogenic factors, such as vascular endothelial growth factor (VEGF) and plateletderived growth factor subunit B (PDGFB), to increase endothelial cell proliferation and vascular densities. This results in vessels and channels invading from the subchondral bone into the cartilage zones to facilitate endochondral ossification. ${ }^{6,7}$ The increase of angiogenesis recruits more lymphocytes, macrophages and other inflammatory cells. ${ }^{8}$ The subchondral bone, a structural girder and shock absorber, provides mechanical support for the overlying articular cartilage. ${ }^{9,10}$ Therefore, the changes in the subchondral bone, which result in the alteration of the subchondral bone's thickness and flexibility, contribute to cartilage lesions and aggravate pain. ${ }^{3,6}$ The vessels are large in the subchondral bone of anterior cruciate ligament transection (ACLT) mice. ${ }^{3}$ The aberrant bone formation through increased nestinpositive mesenchymal stem cells (MSCs) in the subchondral bone is coupled with angiogenesis. ${ }^{3}$ However, the incomplete understanding of aberrant neovascularization, which contributes to articular cartilage degeneration during osteoarthritis progression suggests that additional modulators have yet to be identified.

Leucine-rich-alpha-2-glycoprotein1 (LRG1) is a new regulator of pathogenic angiogenesis and a novel oncogeneassociated protein. ${ }^{11,12}$ Reports showed that LRG1 has an important role in epithelial-mesenchymal transition and angiogenesis in colorectal cancer; and glioma cell invasion, migration and angiogenesis promotion in the damaged retina. ${ }^{11-13}$ Reports showed that LRG1 promotes angiogenesis by modulating endothelial transforming growth factor b1 (TGF- $\beta$ ) signaling. In the angiogenesis condition, LRG1 modulates TGF- $\beta$ to activate smad1/5 phosphorylation. ${ }^{11}$ LRG1 is a promising therapeutic target for pathogenic angiogenesis-associated disease, such as ocular disease, cancer and atherosclerosis, ${ }^{11}$ but it remains unclear whether LRG1 is increased and plays a role in OA or not.

\footnotetext{
${ }^{1}$ The Key Laboratory of Stem Cell Biology, Institute of Health Sciences, Shanghai Institutes for Biological Sciences (SIBS), Chinese Academy of Sciences (CAS); University of Chinese Academy of Sciences, Shanghai 200031, China and ${ }^{2}$ Shanghai Key Laboratory of Orthopaedic Implant, Department of Orthopaedic Surgery, Shanghai Ninth People's Hospital, Shanghai Jiao Tong University School of Medicine (SJTUSM), Shanghai 200011, China

*Corresponding author: K Dai or X Zhang, The Key Laboratory of Stem Cell Biology, Institute of Health Sciences, Shanghai Institutes for Biological Sciences (SIBS), Chinese Academy of Sciences (CAS), 320 Yueyang Road, Shanghai 200031, China. Tel: +8621-54923338; Fax: +8621-54923338; E-mail: krdai@163.com or xlzhang@sibs.ac.cn

Received 07.12.16; revised 14.2.17; accepted 20.2.17; Edited by Y Shi
} 
a

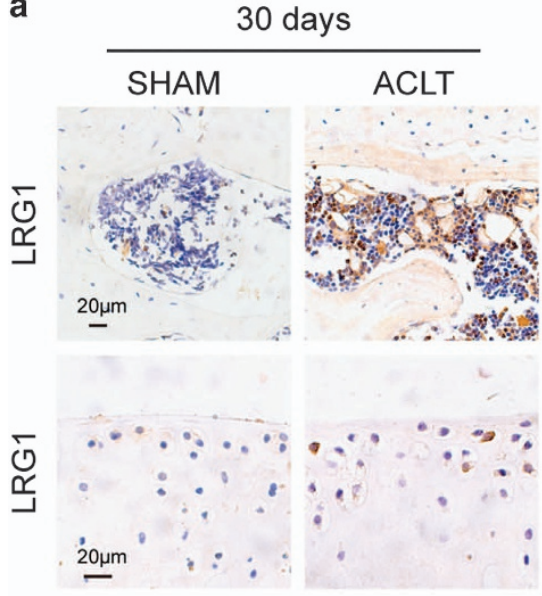

ตั
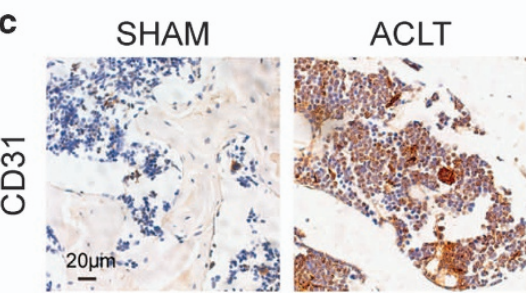

b

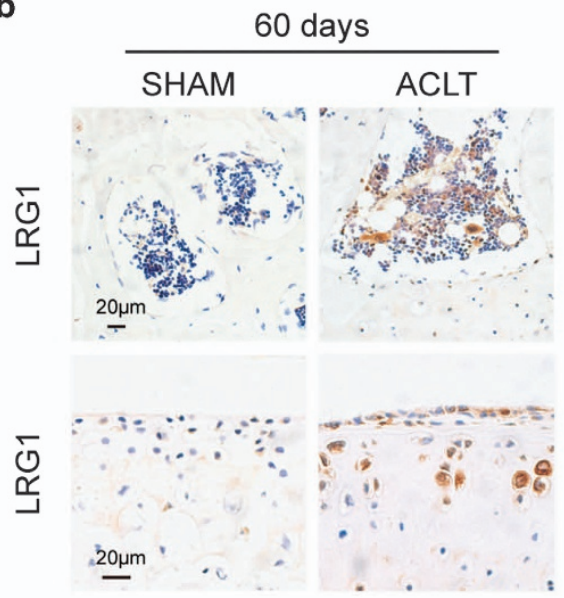

d
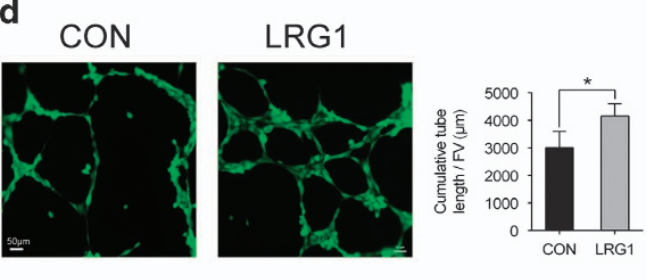

Figure 1 LRG1 was upregulated in the subchondral bone and articular cartilage and associated with angiogenesis in ACLT mice. (a and $\mathbf{b})$ Immunohistochemical analysis results of LRG1 in mouse tibial subchondral bone (top) and articular cartilage (bottom) collected 30 days after ACLT surgery (a) and collected 60 days after ACLT surgery (b); $n=5$ per group. Scale bars, $20 \mu \mathrm{m}$. (c) Immunohistochemical analysis of CD31 in mouse tibial subchondral bone; $n=5$ per group. Scale bars, $20 \mu \mathrm{m}$. (d) Matrigel tube-formation assay images (left) and quantitative analysis of cumulative tube length (right). Scale bar, $50 \mu \mathrm{m}$. Values are given as means \pm S.D. (three independent experiments), ${ }^{*} P<0.05$

Tumor necrosis factor- $a($ TNF- $a)$ is one of the main proinflammatory cytokines involved in $\mathrm{OA}$ pathogenesis. TNF- $a$ stimulates the release of matrix metalloproteinase-1 (MMP-1), MMP-3 and MMP13 and suppresses proteoglycan and type II collagen synthesis in OA. ${ }^{2}$ TNF- $a$ is also a stimulus for the local release of angiogenic substances, such as VEGF, basic fibroblast growth factor (bFGF) and PDGFB, which promote the proliferation and migration of endothelial cells. 8,14 Whether TNF- $a$ affects LRG1 secretion is not known. The small molecule Lenalidomide (LEN) is an inhibitor of TNF- $a$ production. ${ }^{15}$ LEN has shown therapeutic promises in clinical trials for myeloma in consideration of its antiangiogenic and anti-inflammatory effects. ${ }^{16-18}$ Other researches have also focused on its effects in solid tumors, ${ }^{19,20}$ but little is known about its treatment effects on OA.

In this study, the role of LRG1 in the subchondral bone and articular cartilage during $\mathrm{OA}$ progression was investigated. We found that LRG1 was elevated in the subchondral bone and articular cartilage in a mouse OA model. LRG1 promoted the angiogenesis of endothelial cells and the migration of MSC coupling with bone formation. Furthermore, we demonstrated that TNF- $a$ stimulated LRG1 secretion and found the possible signaling pathway involved. Finally, we found that the inhibition of TNF- $a$ production by LEN reduced LRG1 expression, attenuated the pathological changes of subchondral bone and alleviated the degeneration of articular cartilage compared with vehicle-treated groups in the OA models. Our experimental data provide the following: (i) novel molecular mechanisms of OA, suggesting the role of LRG1 that is induced by TNF- $a$ and (ii) a novel potential preventive treatment drug for $\mathrm{OA}$.

\section{Results}

The expression of LRG1 in the subchondral bone and articular cartilage of ACLT mice. Angiogenesis is essential in the progression of $O A$, and we wondered whether LRG1, a new regulator of pathogenic angiogenesis, has an important role in OA. We examined LRG1 expression by immunohistochemistry. We found that LRG1 was upregulated in the subchondral bone in ACLT mice compared with shamoperated mice at 30 and 60 days after operation (Figures $1 \mathrm{a}$ and $\mathrm{b}$ ). We also found that LRG1 significantly upregulated in the articular cartilage 60 days after operation and slightly upregulated 30 days after operation (Figures 1a and b) The number of CD31-positive endothelial cells was larger in the subchondral bone of ACLT mice (Figure 1c). The effectiveness of promoting angiogenesis was verified by HUVEC tube-formation assay in vitro. The LRG1-adding groups showed an enhanced ability to form capillary-like structures compared with vehicle groups (Figure 1d). These results suggested that LRG1 promoted angiogenesis in the subchondral bone of ACLT mice.

The effect of LRG1 on MSC migration. We found that immunostaining for nestin revealed a significantly larger number of nestin-positive cells in the subchondral bone of 
a

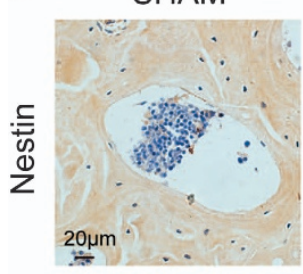

ACLT

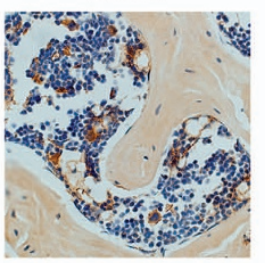

C

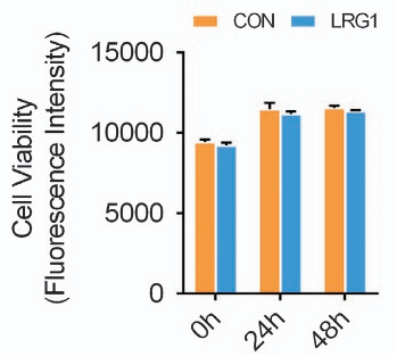

b

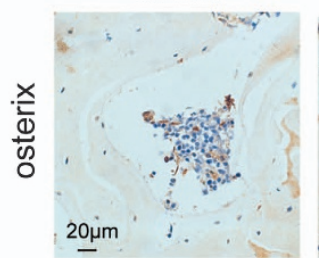

ACLT

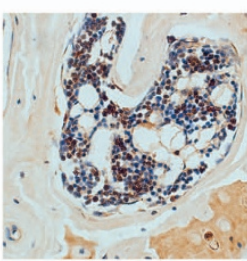

d
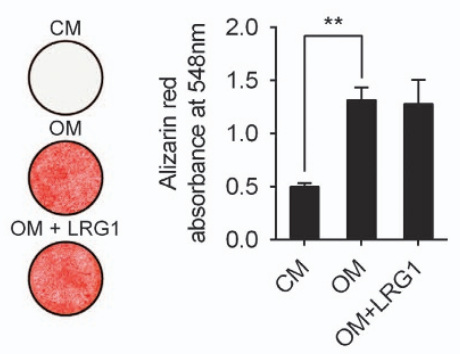
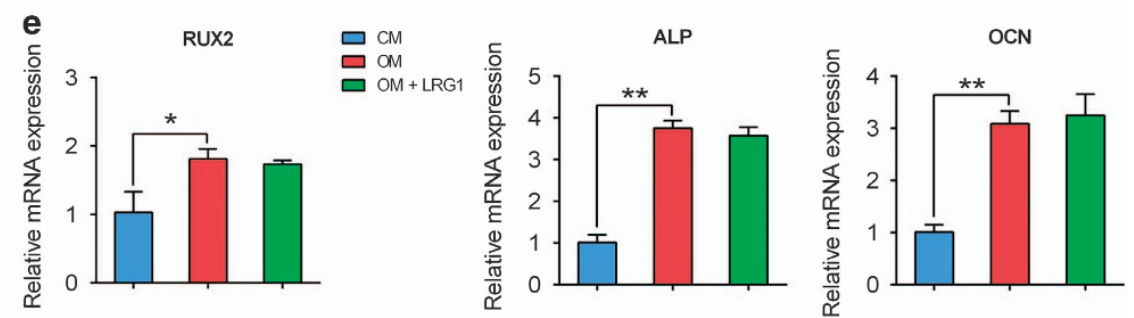

Figure 2 LRG1 had no direct effect on osteogenesis. (a and $\mathbf{b}$ ) Immunohistochemical analysis of nestin (a) and osterix (b) in mouse tibial subchondral bone collected 30 days after ACLT surgery, $n=5$ per group. Scale bars, $20 \mu \mathrm{m}$. (c) The cell proliferation was determined in hBMMSCs by PrestoBlue. (d) Alizarin Red S staining (left) of cultured hBMMSCs after treatment with osteogenic medium plus LRG1. Quantitative of Alizarin Red S staining showed the levels of mineralization (right). CM, control medium; OM, osteogenesis medium; OM+LRG1, osteogenesis medium plus LRG1. (e) Relative expression levels of osteoblast markers in hBMMSCs indicated in d were quantified by RT-PCR. All values are given as means \pm S.D. (three independent experiments); ${ }^{*} P<0.05,{ }^{* \star} P<0.01$

ACLT mice compared with sham-operated groups (Figure 2a). Nestin is supposed to be expressed primarily in adult bone marrow MSCs. ${ }^{21,22}$ Once committed to the osteoblast lineage, MSCs express osterix that is essential for osteoblastogenesis. The number of osterix-positive cells was higher in the subchondral bone of ACLT mice compared with sham-operated controls (Figure $2 \mathrm{~b}$ ), which indicated that the nestin-positive MSCs might participate in the de novo bone formation through osteoblastic differentiation. Thus, we explored whether LRG1 contributes to the increased nestinpositive MSCs in ACLT mice. To confirm this, we cultured hBMMSCs exposed to LRG1 to analyze cell proliferation, but LRG1-treated hBMMSCs had no significant difference with the controls in proliferation (Figure 2c). Then, we induced osteogenic differentiation of the human bone marrow mesenchymal stem cells (hBMMSCs) to find whether LRG1 promotes osteogenesis or not. However, LRG1 exerted no direct promotion effects on osteogenesis (Figures $2 \mathrm{~d}$ and e). However, LRG1 induced significant hBMMSC migration relative to the control (Figures $3 a$ and $b$ ). Mitogen-activated protein kinases (MAPKs) are known to have important roles in cell migration. ${ }^{23,24}$ We assessed their roles in LRG1induced hBMMSC migration. Western blot analysis results showed that LRG1 significantly induced phosphorylation of p38 in hBMMSCs as shown in Figure 3c. To confirm that
LRG1-induced migration was mediated through p38 pathway, the phosphorylation of p38 was blocked by p38 MAPK inhibitor in the Transwell assays. The inhibition of p38 pathway was sufficient to block LRG1-induced migration of hBMMSCs (Figures $3 d$ and e). The results indicated that instead of promoting osteogenesis directly, LRG1 promoted hBMMSC migration through p38 signaling to contribute in the osteogenesis.

The effect of TNF- $\alpha$ on LRG1 secretion. Interleukin- $1 \beta$ (IL-1 $\beta)$, TNF- $\alpha$, IL- 6 and IL-17are considered to be the main proinflammatory cytokines involved in the pathophysiology of $\mathrm{OA}^{2}$ These proinflammatory cytokines upregulate angiogenic factors to stimulate angiogenesis. Therefore, we investigated the influence of these proinflammatory cytokines on LRG1 secretion in human umbilical vein endothelial cells (HUVECs). To this end, we assessed the changes in the mRNA expression of LRG1 in HUVECs treated with recombinant IL-1 $\beta$, TNF- $a$, IL-6 and IL-17 (10 or $50 \mathrm{ng} / \mathrm{ml}$ ). Among the tested cytokines, TNF- $\alpha$-treated groups significantly increased the expression of LRG1 in both dosages, whereas IL- $1 \beta$ and IL- 6 had no obvious effects on LRG1 expression. IL-17 slightly upregulated LRG1 in $50 \mathrm{ng} / \mathrm{ml}$ not $10 \mathrm{ng} / \mathrm{ml}$ (Figure 4a). We further analyzed the levels of LRG1 protein in HUVECs treated with different dosages of 
a

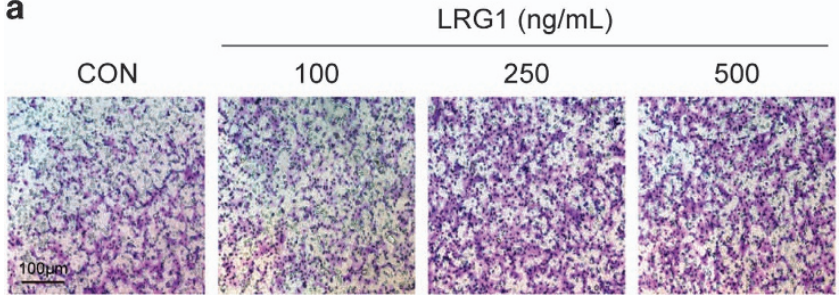

b

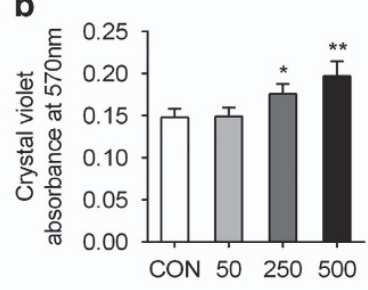

c

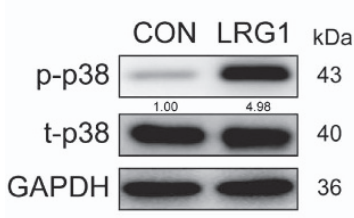

\begin{abstract}
d
\end{abstract}

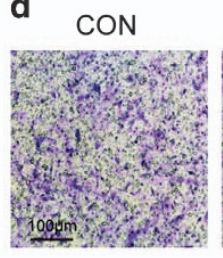

LRG1

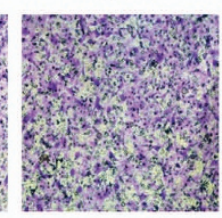

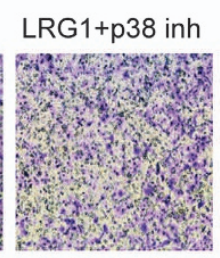

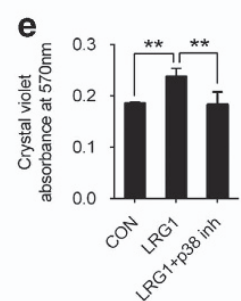

Figure 3 LRG1 induced hBMMSCs migration. (a and $\mathbf{b})$ Transwell assay images (a) and quantitative analysis (b) for the migration of hBMMSCs by using mediums plus different concentrations of LRG1. Scale bar, $100 \mu \mathrm{m}$. (c) Western blots of the p38 phosphorylation in hBMMSCs treated with LRG1 for 15 min. The relative expression levels of protein are shown at the bottom of the bands as normalized by the total p38 level. (d and e) Transwell assay images (d) and quantitative analysis (e) for the migration of hBMMSCs that were preincubated with vehicle or p38 inhibitor (inh p38) by using indicated mediums. Scale bar, $100 \mu \mathrm{m}$. All values are given as means \pm S.D. (three independent experiments); ${ }^{*} P<0.05,{ }^{* *} P<0.01$

recombinant TNF- $a(10-50 \mathrm{ng} / \mathrm{ml})$ by western blot. As expected, the TNF- $a$ increased LRG1 expression (Figure 4b). To affirm that TNF- $a$ induces the secretion of LRG1 in HUVECs to promote angiogenesis and MSC migration, we used siRNA to inhibit LRG1 expression in HUVECs (Figure 4c). We collected the supernatants of siLRG1 or siNC-treated HUVECs that were induced with TNF- $a$. Then, we used the supernatants to induce HUVEC tube formation and hBMMSC migration. The knockdown of LRG1 expression led to a significant decrease in HUVEC tube formation and hBMMSC migration (Figures $4 d$ and e). The activation of MAPKs and NF- $K \mathrm{~B}$ is known to regulate TNF- $a$-induced gene expression. Western blot analysis showed that TNF- $a$ induced phosphorylation of p38 and p65 in HUVECs (Figure 4f). To verify the involvement of these signaling in upregulated LRG1 responses to TNF- $a$, we pretreated HUVECs with the MAPKs or NF- $K B$ inhibitor independently. We found that p38 MAPK inhibitor and NF- $k B$ inhibitor reduced TNF- $a$-induced LRG1 mRNA expression (Figure 4g). Consistently, western blot analysis showed that TNF- $a$ induced LRG1 protein level was reduced by p38 MAPK and NF- $K B$ inhibitors (Figure $4 \mathrm{~h}$ ). These data indicated that TNF- $a$ induced the expression of LRG1 through p38 and $\mathrm{NF}-\kappa \mathrm{B}$ signaling to promote angiogenesis and MSC migration.

Inhibition of TNF- $a$ and LRG1 by LEN in OA. LEN is an inhibitor of TNF- $a$ production. ${ }^{15}$ We are interested in whether the inhibition of TNF- $a$ secretion by LEN reduces LRG1 expression, and diminishes angiogenesis and aberrant bone formation to attenuates OA. So, we injected LEN (50 mg per $\mathrm{kg}$ body weight) intraperitoneally daily in ACLT mice. The immunostaining of the subchondral bone showed that TNF- $a$ was higher in ACLT mice relative to sham-operated controls, and this effect was prevented by LEN treatment (Figure 5a). Consistently, the expression of LRG1 was significantly higher in the subchondral bone of ACLT mice relative to shamoperated controls, and this effect was inhibited by the injection of LEN (Figure 5a). Moreover, the number of CD31-positive endothelial progenitors was significantly larger in the subchondral bone of ACLT mice than that of shamoperated controls, and this effect was reduced by LEN treatment, suggesting reduced angiogenesis (Figure 5a).

We found by immunostaining that the increased number of nestin-positive MSCs in the subchondral bone of ACLT mice relative to sham-operated controls was reduced by the injection of LEN (Figure 5b). The number of osterix-positive osteoprogenitors was reduced by the injection of LEN in ACLT mice (Figure $5 b$ ), suggesting a decreased aberrant bone formation.

The degeneration of articular cartilage was attenuated by LEN administration for 2 months after surgery as shown in Figure $5 \mathrm{c}$. A time point is usually used for the severity analysis in mechanical destabilized OA mice models. ${ }^{25}$ We quantified the protective effect of LEN on articular cartilage using the Osteoarthritis Research Society International (OARSI) system $^{26}$ (Figure 5d).The immunostaining of the articular cartilage showed that the number of MMP13-positive chondrocytes was smaller in the LEN-treated than the vehicletreated ACLT mice, reflecting the protection from articular cartilage degeneration (Figure 5e). The LRG1-positive chondrocytes were reduced in the LEN-treated than the vehicletreated ACLT mice (Figure 5e). Collectively, LEN treatment attenuated the pathological changes in the subchondral bone and led to less degeneration of articular cartilage compared with vehicle-treated ACLT mice.

\section{Discussion}

Angiogenesis is important in OA development. During OA, angiogenesis is increased in the subchondral bone, which leads to vascularization and lesion formation in the 
a

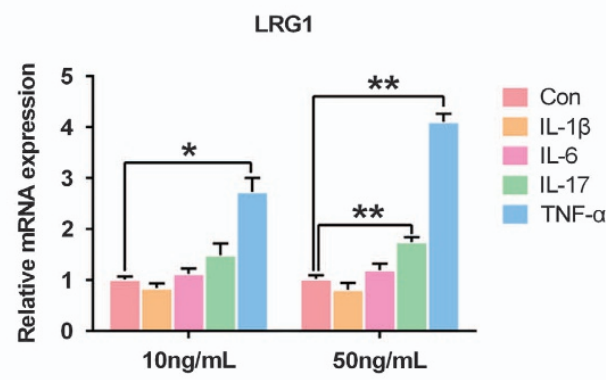

d

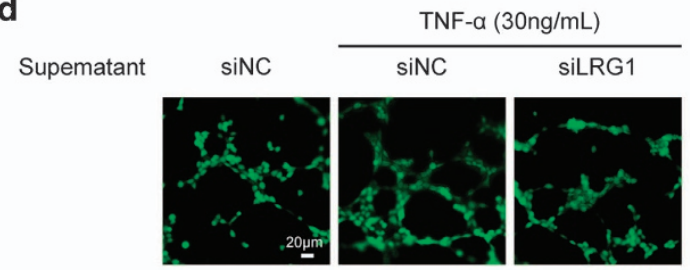

e

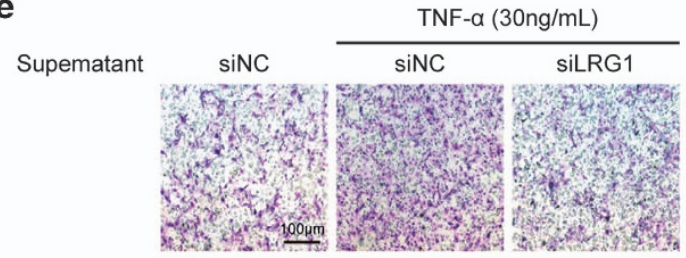

f

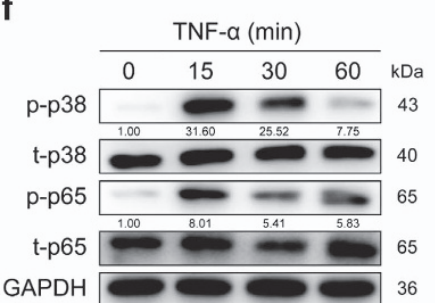

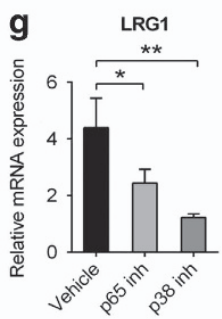

b

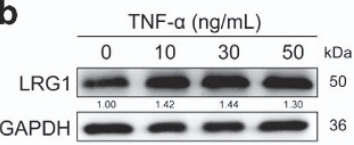

C
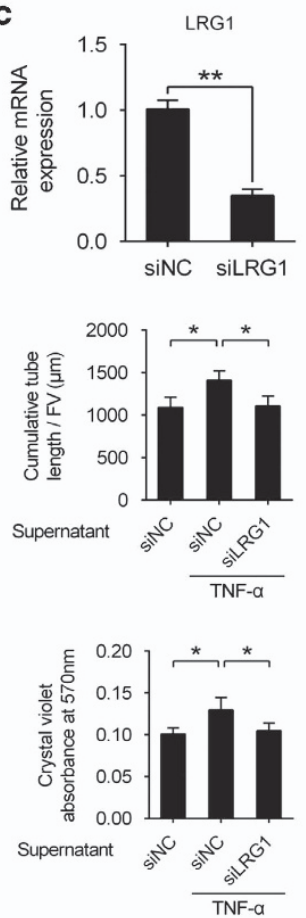

h

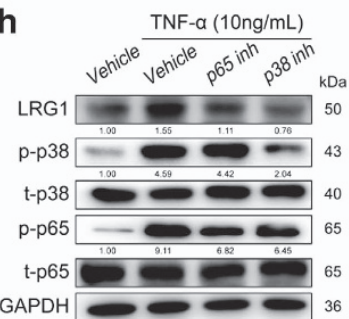

Figure 4 TNF- $\alpha$ induced LRG1 secretion in HUVECs through $p 38$ and p65 signaling. (a) RT-PCR analysis of LRG1 in HUVECs, stimulated with IL-1 $\beta$, TNF- $\alpha$, IL-6 and IL-17 (10 or $50 \mathrm{ng} / \mathrm{ml}$ ). (b) Western blot analysis of LRG1 in HUVECs stimulated with TNF- $\alpha$ in different concentrations. The relative expression levels of protein are shown at the bottom of the bands as normalized by the GAPDH level. (c) Knockdown of LRG1 expression in HUVECs by siRNA. (d) Matrigel tube-formation assay of HUVECs after treatment with supernatants, which were collected from siLRG1 or siNC transfected HUVEC cultures in the presence of TNF- $\alpha$. Images (left) and quantitative analysis of cumulative tube length (right). Scale bar, $20 \mu \mathrm{m}$. (e) Transwell assays of hBMMSCs after treatment with supernatants, which were collected from siLRG1 or siNC transfected HUVEC cultures in the presence of TNF- $\alpha$. Images (left) and quantitative analysis (right). Scale bar, $100 \mu \mathrm{m}$. (f) Western blot analysis of the phosphorylation of p38 and p65 in HUVECs treated with TNF- $\alpha$ at different times. The relative expression levels of protein are shown at the bottom of the bands as normalized by the total p38 and p65 levels, respectively. (g and $\mathbf{h}$ ) RT-PCR (g) or western blot analysis of LRG1 and phosphorylation of p38 and p65 (h) in HUVECs stimulated with TNF- $\alpha$ and pretreated or not with p38 inhibitor (p38 inh) and p65 inhibitor (p65 inh). The relative expression levels of protein are shown at the bottom of the bands. LRG1 level is normalized by the GAPDH level, and the phosphorylation of p38 and p65 levels are normalized by the total p38 and p65 levels, respectively. All values are given as means \pm S.D. (three independent experiments); ${ }^{*} P<0.05,{ }^{* \star} P<0.01$

osteoarthritic cartilage..$^{3,4,6,7}$ The cells within the subchondral bone express angiogenic factors (such as VEGF and PDGFB) that increase endothelial cell proliferation and vascular densities in OA.4,6,27 Recently, a novel angiogenic factor, LRG1, was identified as a regulator of pathogenic angiogenesis. ${ }^{11}$ However, whether LRG1 has an important role in OA remains unclear. Thus, we hypothesized that LRG1 promoted the angiogenesis in the subchondral bone. Consistently, we found that LRG1 was upregulated in the subchondral bone marrow of ACLT mice. The chondrocytes also express some angiogenic factors that promote vessel and channel invasion from the subchondral bone into the cartilage zones. ${ }^{4,6,27}$ We further found that LRG1 was also upregulated in the articular cartilage of ACLT mice. Evidence has shown that blood vessels and the number of CD31+ endothelial progenitor cells in the subchondral bone were significantly higher in ACLT mice relative to sham mice. ${ }^{3}$ Consistently, we found that the number of CD31-positive cells in the subchondral bone marrow was larger in ACLT mice, revealing elevated angiogenesis. We confirmed that LRG1 enhanced the tube 

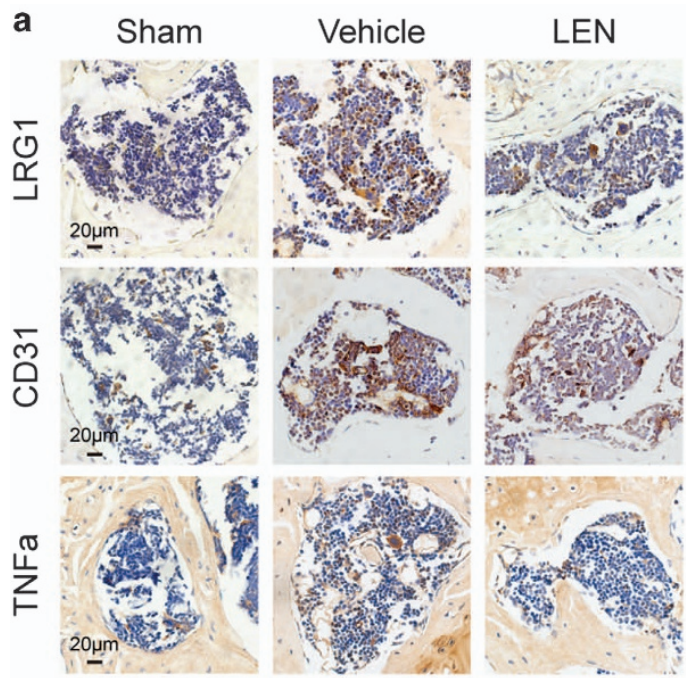

c

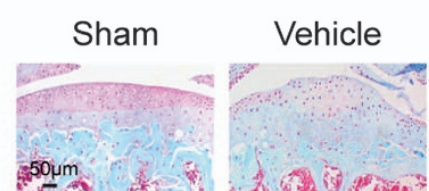

d

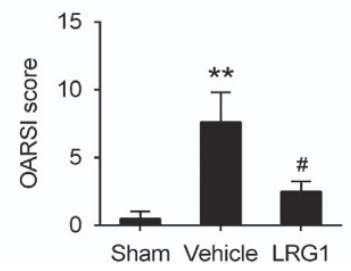

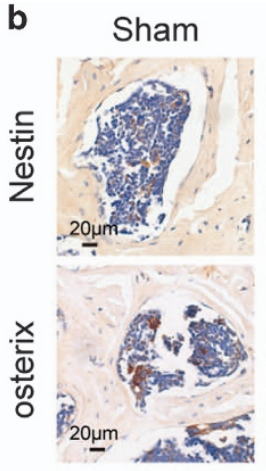
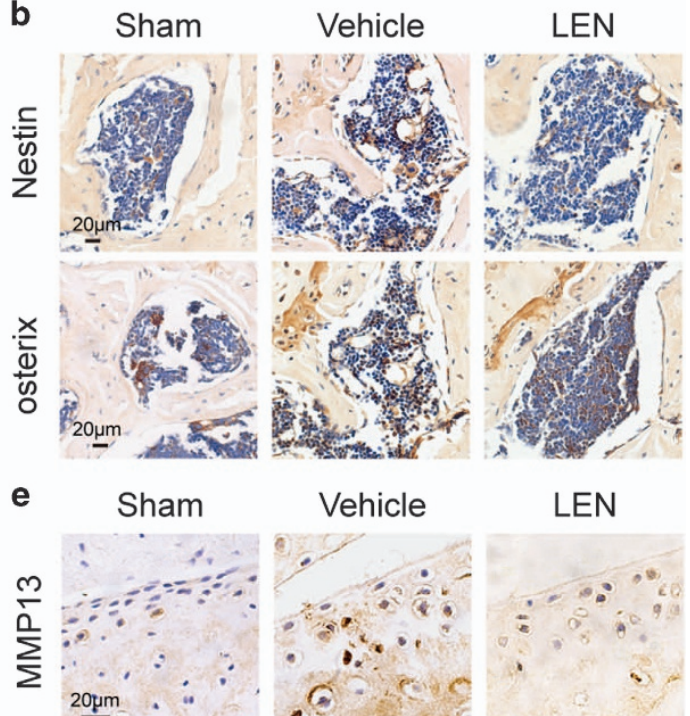

Vehicle

LEN

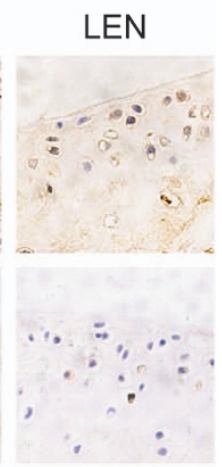

Figure 5 LEN, a TNF- $\alpha$ production inhibitor, reduced LRG1 expression, alleviated the changes in the subchondral bone, and attenuated articular cartilage degeneration in ACLT. (a and b) Immunohistochemical analysis of LRG1 (top, a), CD31 (middle, a), TNF- $\alpha$ (bottom, a), nestin (Top, $\mathbf{b}$ ) and osterix (bottom, $\mathbf{b}$ ) in tibial subchondral bone harvested 30 days after sham operation (sham), ACLT operation and treatment with vehicle (vehicle) or ACLT operation and treatment with LEN (LEN); $n=5$ per group. Scale bars, $20 \mu \mathrm{m}$. (c) Safranin $O$ and fast green staining in the articular cartilage of mice 60 days after surgery; $n=5$ per group. Scale bars, $50 \mu \mathrm{m}$. (d) OARSI scores in c. (e) Immunohistochemical analysis of MMP13 (top) and LRG1 (bottom) in the articular cartilage of mice 30 days after surgery; $n=5$ per group. Scale bars, $20 \mu \mathrm{m}$. All values are given as means \pm S.D. ${ }^{* *} P<0.01$ compared with the sham-operated group; ${ }^{\#} P<0.05$ compared with the vehicle-treated ACLT group

formation of HUVECs in vitro at different dosage. We found that LRG1 promoted tube-formation well at $500 \mathrm{ng} / \mathrm{ml}$, which demonstrated the angiogenic effect. Therefore, these data suggest that LRG1 is elevated and promoted angiogenesis in $\mathrm{OA}$.

$\mathrm{OA}$ is developing along with the accretion of mesenchymal progenitor cells in the joint tissues and synovial fluids. ${ }^{3,28,29}$ Consistently, mechanical destabilized joints exerted a large number of nestin-positive MSCs and osterix-positive osteoprogenitors in the subchondral bone marrow in ACLT mouse models in our study. The altered mechanical loading leads to aberrant bone formation by in situ commitment of osteoprogenitors rather than normal bone remodeling by osteoblasts and their progenitors at the resorption site on the bone surface. ${ }^{3,30}$ In this study, we tested the ability of LRG1 to recruit hBMMSCs by Transwell assay. We found that LRG1 induced significant hBMMSCs migration. The enhanced migration was inhibited by the blockage of p38 signaling pathway. The ability of LRG1 to recruit MSCs may contribute to the in situ aberrant bone formation. The changes in the subchondral bone contribute to cartilage lesions and aggravate pain. ${ }^{31}$ Bone formation is often coupled with angiogenesis. The formation of new blood vessels is required for supplying nutrients, oxygen, growth factors and cytokines as required for bone tissue. ${ }^{32-34}$ Therefore, the elevated angiogenesis in the subchondral bone marrow in ACLT mice is convenient for the aberrant bone formation, and some angiogenic growth factors secreted by fibrovascular tissue stimulate the migration of MSCs. ${ }^{35,36}$ Thus, we suggest that LRG1 has an important role in the angiogenesis coupling with aberrant bone formation by recruiting MSCs in the subchondral bone in OA.

Proinflammatory cytokines have important roles in the pathophysiology of $\mathrm{OA}$, and many of them upregulate angiogenic factors to stimulate angiogenesis. ${ }^{14,37-39}$ Thus, we tested the effects of main proinflammatory cytokines IL-1 $\beta$, TNF- $a$, IL- 6 and IL-17 on LRG1 expression in HUVECs. Our results showed that IL-1 $\beta$, and IL- 6 had no obvious effects on LRG1 expression and IL-17 at $50 \mathrm{ng} / \mathrm{ml}$ mildly upregulated LRG1. However, TNF- $a$ significantly increased the expression 
of LRG1 in HUVECs. TNF- $a$ has been shown to promote cell proliferation and angiogenesis. ${ }^{40}$ Thus, we hypothesized that TNF- $a$ induces the secretion of LRG1 in HUVECs to promote angiogenesis and MSC migration. Consistently, the supernatants of TNF- $a$-stimulated HUVECs promoted angiogenesis and MSC migration, and this effect was prevented by the supernatants of siLRG1-treated HUVECs. MAPK and NF- $\kappa \mathrm{B}$ signaling pathway are typically involved in the downstream function of TNF- $a .^{40,41}$ Consistent with this, our study found that the inhibition of the kinases p38 and p65 reduced TNF- $a$ induced LRG1 secretion, suggesting that these molecules are implicated in the LRG1 expression in HUVECs. Results suggest that TNF- $a$ is a proinflammatory cytokine of inducing LRG1 expression in OA pathogenesis.

The small molecule LEN is an inhibitor of TNF- $a$ production. ${ }^{15}$ We hypothesized that the inhibition of TNF- $a$ production by LEN reduced TNF- $a$-induced LRG1 secretion, and alleviated angiogenesis and aberrant bone formation to attenuate OA. To test our hypothesis, we injected LEN intraperitoneally daily in ACLT mice. We showed that LEN suppressed the expression of TNF- $a$ and LRG1 and reduced the number of CD31-positive cells in the subchondral bone, suggesting diminished angiogenesis. It is known that the elevated vessels invade from the subchondral bone into the cartilage zones to facilitates endochondral ossification in OA. ${ }^{6,7}$ The decreased angiogenesis probably alleviates the process. The reduction of nestin-positive MSCs and osterixpositive osteoprogenitors in the LEN-injected mice revealed less MSCs migration and aberrant bone formation. The decreased aberrant bone formation in the subchondral bone marrow probably alleviates the degeneration of articular cartilage. Consistently, it showed alleviated articular cartilage degeneration in LEN-treated mice. LEN has shown therapeutic promises in clinical trials for the treatment of multiple myeloma in consideration of its antiangiogenic and antiinflammatory effects. ${ }^{16-20}$ Recently, some treatment strategies for $\mathrm{OA}$ are aimed at anti-TNF- $a$ and antiangiogenesis. ${ }^{42,43}$ We expect that treatment with LEN is a potential approach for ameliorating $\mathrm{OA}$.

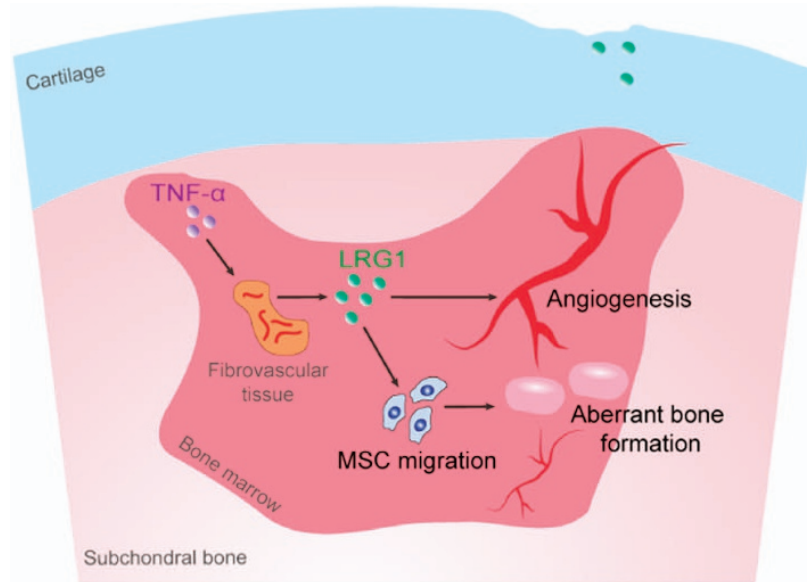

Figure 6 Proposed role of LRG1 in OA. The elevated TNF- $\alpha$ induces LRG1 secretion. LRG1 promotes angiogenesis coupling with de novo bone formation by recruiting MSCs in the subchondral bone of $O A$ joints
In conclusion, during OA progression, the elevated TNF- $a$ is the dominating proinflammatory cytokine that induces LRG1 secretion. LRG1 promotes angiogenesis coupling with de novo bone formation by recruiting MSCs in the subchondral bone of OA joints (Figure 6). LEN is an avenue for OA treatment.

\section{Materials and Methods}

Mice. Male C57BL/6J mice were purchased from Shanghai SLAC Laboratory Animal Co., Ltd. The mice were maintained in a specific pathogen-free animal facility of the Institute of Health Sciences, Shanghai Institute for Biological Sciences and Shanghai Jiao Tong University School of Medicine.

Mice aged 8 weeks were anesthetized, and then transected the anterior cruciate ligament surgically to induce mechanical destabilized $O A$ on the right knee. Sham operations were made a capsular incision on the independent mice. The operated mice were killed at 30 or 60 days after surgery ( $n=5$ per group). For the therapeutic experiment 3 days before surgery, we injected LEN (Selleck, Houston, TX, USA; $50 \mathrm{mg}$ per $\mathrm{kg}$ body weight) or the equivalent volume of vehicle (DMSO and PBS) intraperitoneally daily for 30 or 60 days. The mice were killed 30 or 60 days after surgery.

All the operations were performed under protocols approved by the Institutional Animal Care and Use Committee of the Institute of Health Sciences, Shanghai Institute for Biological Sciences and Shanghai Jiao Tong University School of Medicine.

Histochemistry and immunohistochemistry. At the time of killing, the knee joints of mice were fixed in $4 \%$ paraformaldehyde, decalcified with $12.5 \%$ EDTA (pH 7.0) and embedded in paraffin. The knee joint sections $(7 \mu \mathrm{m})$ were stained with $\mathrm{H \& E}$ and safranin $\mathrm{O}$ and fast green. For the immunostaining, the sections were incubated with primary antibodies to mouse nestin (1:800; Abcam, Cambridge, MA, USA), osterix (1:1000; Abcam), LRG1 (1:100; Abcam), CD31 (1:100; Abcam), TNF- $\alpha$ (1:100; Abcam) and MMP13 (1:200; Bioworld Technology) overnight at $4^{\circ} \mathrm{C}$. These samples were incubated with goat antirabbit secondary antibodies conjugated with horseradish peroxidase (HRP).

Cell culture. The HUVECs and hBMMSCs were obtained from the Institute of Health Sciences, Shanghai Institute for Biological Sciences and Shanghai Jiao Tong University School of Medicine.

For cytokine stimulation analysis, HUVECs were cultured in a medium to full confluence, stimulated or not with different dosages of the indicated cytokines (IL-1 $\beta$, TNF- $\alpha$, IL-6 and IL-17; all from PeproTech, Rocky Hill, New Jersey, USA), and treated or not with different inhibitors: P38 MAPK inhibitor (SB203580, $10 \mu \mathrm{M}$; Selleckchem, Houston, TX, USA) and NF- $\kappa$ B inhibitor (BAY 11-7082, $10 \mu \mathrm{m}$; Beyotime Co., Jiangsu, China).

HUVEC tube-formation assay. Growth factor reduced Matrigel (BD Biosciences) was plated in 96-well culture plates and incubated at $37^{\circ} \mathrm{C}$ to polymerize for $30 \mathrm{~min}$. HUVECs $\left(2 \times 10^{4}\right.$ cells/well) were seeded on polymerized Matrigel in the plates. The cells were cultured with medium alone or plus LRG1 $\left(500 \mathrm{ng} / \mathrm{ml}\right.$; Abcam). After incubation at $37^{\circ} \mathrm{C}$ for $4-6 \mathrm{~h}$, we stained the cells with calcein and observed the tube formation by microscopy. The cumulative tube lengths were measured.

For the supernatant assay, HUVECs were transfected with small interfering RNA (siRNA; Shanghai GenePharma Co.,Ltd, Shanghai, China) for LRG1 (siLRG1) or scramble siRNA (siNC) and stimulated with TNF- $\alpha$ ( $30 \mathrm{ng} / \mathrm{ml}$, PeproTech). Then the supernatants were collected after 3 days of stimulation. The supernatants combined with high-glucose DMEM medium were used to culture the HUVECs in the tubeformation assay. The siRNA for LRG1 was transfected using Lipofectamine 2000 (Invitrogen, Carlsbad CA, USA) as described by the manufacturer. The target sequence of LRG1 mRNA was 5'-GCAAUUAGAACGGCUACAU-3', and that of scramble siRNA (siNC) was 5'-UUCUCCGAACGUGUCACGU-3'.

The transwell assays of hBMMSCs. Cell migration was measured by using the 48-well Transwell plates (Millipore, Darmstadt, Germany) with $8 \mu \mathrm{m}$ pore filters. A total of $5.5 \times 10^{4} /$ well hBMMSCs were seeded in the upper chambers and then incubated them with medium alone or plus different concentrations of LRG1 in the lower chambers for $2-3 \mathrm{~h}$ at $37{ }^{\circ} \mathrm{C}$ in a $5 \% \mathrm{CO}_{2}$-filled incubator. After incubation, the cells were fixed with $4 \%$ paraformaldehyde for $30 \mathrm{~min}$. The cells on 
the upper surface of each filter were removed with cotton swabs. The cells that had migrated to the lower surface were stained with $0.5 \%$ crystal violet (Sigma-Aldrich, St. Louis, MO, USA) and photographed. Finally, the stained chambers were eluted with $33 \%$ acetic acid solution and quantified the eluent by using a TecanSafire2 microplate reader (Tecan, Durham, NC, USA) by absorbance at $570 \mathrm{~nm}$. In the inhibitor assay, the hBMMSCs were preincubated with vehicle or p38 MAPK inhibitor (SB203580, $30 \mu \mathrm{M}$, Selleckchem).

For the supernatant assay, the supernatants were collected as described above. The supernatants combined with high-glucose DMEM medium were added to the lower chambers.

Osteogenic differentiation assay. The hBMMSCs were cultured in osteogenic medium containing $100 \mathrm{nM}$ dexamethasone, $50 \mu \mathrm{M}$ ascorbic acid, and $10 \mathrm{~mm} \beta$-glycerophosphate (all from Sigma-Aldrich). LRG1 (500 ng/ml; Abcam) was added. The medium was changed every 3 days. To assess the osteogenic differentiation, these cultures were stained with Alizarin Red S (Sigma-Aldrich). Finally, the calcium precipitates were dissolved in $0.1 \mathrm{~N}$ sodium hydroxide and quantified by using a TecanSafire2 microplate reader (Tecan) by absorbance at $548 \mathrm{~nm}$.

Cell proliferation assay. The hBMMSCs were seeded in 48-well plates for $24 \mathrm{~h}$ and then treated with LRG1 $(500 \mathrm{ng} / \mathrm{ml})$ for 0,24 and $48 \mathrm{~h}$. The cell viability was measured by using PrestoBlue Cell Viability Reagent (Thermo Scientific, Waltham, MA, USA) and quantified by using a TecanSafire2 microplate reader (Tecan) by excitation at $560 \mathrm{~nm}$ and emission at $590 \mathrm{~nm}$ according to the manufacturer's instructions.

RNA isolation and real-time polymerase chain reaction. The total RNA was isolated by using TRIZOL reagent (Invitrogen, Mulgrave, Australia). The RNA was reversely transcribed into cDNA by RevertAid Reverse Transcriptase (EP0442; Thermo Scientific) according to the manufacturer's instructions. Real-time polymerase chain reaction (RT-PCR) was performed with SYBRPremix EX Taq (Takara, Dalian, China) by using ViiATM 7 (Life Technologies, Carlsbad, CA, USA). Primers used in this study were the following: LRG1, 5'-GGACACCCTGGTATT GAAAGAAA-3' and 5'-TAGCCGTTCTAATTGCAGCGG-3'.

Western blot analysis. Western blot analysis was conducted by a previously described protocol. ${ }^{44}$ Proteins were separated by SDS-PAGE and transferred onto PVDF membranes (Millipore). Blots were probed with primary antibodies to LRG1 (1:500; Abcam), p38, phosphor-p38, p65, phosphor-p65 (1:1000; all from Cell Signaling Technology, Danvers, MA, USA), and GAPDH (1:5000; Kangcheng, Shanghai, China) overnight at $4{ }^{\circ} \mathrm{C}$. These blots were then incubated with goat antirabbit secondary antibodies conjugated with HRP and visualized by using the enhanced chemiluminescence detection system (Millipore).

Statistical analysis. Two-tailed Student's t-test and analysis of variance (ANOVA) were conducted to assess statistical significance. $P<0.05$ was considered statistically significant. Results are presented as the mean \pm S.D.

\section{Conflict of Interest}

The authors declare no conflict of interest.

Acknowledgements. This work was supported by grants from the Ministry of Science and Technology of China (No.2015DFG32200), National Natural Science Foundation of China (No. 81401844 and 81572123), Science and Technology Commission of Shanghai (No. 15411951100, 16ZR1441700, 16430723500), Shanghai Municipal Commission of Health and Family Planning (No. 2013ZYJB0501) and Shanghai Municipal Education Commission-Gaofeng Clinical Medicine Grant Support (20161314).

1. Hootman JM, Helmick CG. Projections of US prevalence of arthritis and associated activity limitations. Arthritis Rheum 2006; 54: 226-229.

2. Kapoor M, Martel-Pelletier J, Lajeunesse D, Pelletier JP, Fahmi H. Role of proinflammatory cytokines in the pathophysiology of osteoarthritis. Nat Rev Rheumatol 2011; 7: 33-42.

3. Zhen G, Wen C, Jia X, Li Y, Crane JL, Mears SC et al. Inhibition of TGF-beta signaling in mesenchymal stem cells of subchondral bone attenuates osteoarthritis. Nat Med 2013; 19: 704-712
4. Mapp PI, Walsh DA. Mechanisms and targets of angiogenesis and nerve growth in osteoarthritis. Nat Rev Rheumatol 2012; 8: 390-398.

5. Andia I, Maffulli N. Platelet-rich plasma for managing pain and inflammation in osteoarthritis. Nat Rev Rheumatol 2013; 9: 721-730.

6. Suri S, Walsh DA. Osteochondral alterations in osteoarthritis. Bone 2012; 51: 204-211.

7. Yuan XL, Meng HY, Wang YC, Peng J, Guo QY, Wang AY et al. Bone-cartilage interface crosstalk in osteoarthritis: potential pathways and future therapeutic strategies. Osteoarthritis Cartilage 2014; 22: 1077-1089.

8. Gravallese EM, Pettit AR, Lee R, Madore R, Manning C, Tsay A et al. Angiopoietin-1 is expressed in the synovium of patients with rheumatoid arthritis and is induced by tumour necrosis factor alpha. Ann Rheum Dis 2003; 62: 100-107.

9. Madry H, van Dijk CN, Mueller-Gerbl M. The basic science of the subchondral bone. Knee Surg Sports Traumatol Arthrosc 2010; 18: 419-433.

10. Lories RJ, Luyten FP. The bone-cartilage unit in osteoarthritis. Nat Rev Rheumatol 2011; 7: $43-49$.

11. Wang $X$, Abraham S, McKenzie JA, Jeffs N, Swire M, Tripathi VB et al. LRG1 promotes angiogenesis by modulating endothelial TGF-beta signalling. Nature 2013; 499: 306-311.

12. Zhang J, Zhu L, Fang J, Ge Z, Li X. LRG1 modulates epithelial-mesenchymal transition and angiogenesis in colorectal cancer via HIF-1alpha activation. J Exp Clin Cancer Res 2016; 35 : 29.

13. Zhong D, He G, Zhao S, Li J, Lang Y, Ye W et al. LRG1 modulates invasion and migration of glioma cell lines through TGF-beta signaling pathway. Acta Histochem 2015; 117: 551-558.

14. Marrelli A, Cipriani P, Liakouli V, Carubbi F, Perricone C, Perricone R et al. Angiogenesis in rheumatoid arthritis: a disease specific process or a common response to chronic inflammation? Autoimmun Rev 2011; 10: 595-598.

15. Muller GW, Chen R, Huang SY, Corral LG, Wong LM, Patterson RT et al. Amino-substituted thalidomide analogs: potent inhibitors of TNF-alpha production. Bioorg Med Chem Lett 1999; 9: $1625-1630$.

16. Richardson PG, Schlossman RL, Weller E, Hideshima T, Mitsiades C, Davies F et al. Immunomodulatory drug CC-5013 overcomes drug resistance and is well tolerated in patients with relapsed multiple myeloma. Blood 2002; 100: 3063-3067.

17. Davies FE, Raje N, Hideshima T, Lentzsch S, Young G, Tai YT et al. Thalidomide and immunomodulatory derivatives augment natural killer cell cytotoxicity in multiple myeloma. Blood 2001; 98: 210-216.

18. Yang B, Yu RL, Chi XH, Lu XC. Lenalidomide treatment for multiple myeloma: systematic review and meta-analysis of randomized controlled trials. PLOS ONE 2013; 8: e64354.

19. Bridoux F, Chen N, Moreau S, Arnulf B, Moumas E, Abraham J et al. Pharmacokinetics, safety, and efficacy of lenalidomide plus dexamethasone in patients with multiple myeloma and renal impairment. Cancer Chemother Pharmacol 2016; 78: 173-182.

20. Leuci V, Maione F, Rotolo R, Giraudo E, Sassi F, Migliardi G et al. Lenalidomide normalizes tumor vessels in colorectal cancer improving chemotherapy activity. J Transl Med 2016; 14: tumor

21. Mendez-Ferrer S, Michurina TV, Ferraro F, Mazloom AR, Macarthur BD, Lira SA et al. Mesenchymal and haematopoietic stem cells form a unique bone marrow niche. Nature 2010; 466: 829-834.

22. Wiese C, Rolletschek A, Kania G, Blyszczuk P, Tarasov KV, Tarasova $Y$ et al. Nestin expression-a property of multi-lineage progenitor cells? Cell Mol Life Sci 2004; 61: 2510-2522.

23. Huang C, Jacobson K, Schaller MD. MAP kinases and cell migration. J Cell Sci 2004; 117: 4619-4628.

24. Tarcic G, Yarden Y. MAP Kinase activation by receptor tyrosine kinases: in control of cell migration. Methods Mol Biol 2010; 661: 125-135.

25. Kamekura S, Hoshi K, Shimoaka T, Chung U, Chikuda H, Yamada T et al. Osteoarthritis development in novel experimental mouse models induced by knee joint instability. Osteoarthritis Cartilage 2005; 13: 632-641.

26. Pritzker KP, Gay S, Jimenez SA, Ostergaard K, Pelletier JP, Revell PA et al. Osteoarthritis cartilage histopathology: grading and staging. Osteoarthritis Cartilage 2006; 14: 13-29.

27. Franses RE, McWilliams DF, Mapp PI, Walsh DA. Osteochondral angiogenesis and increased protease inhibitor expression in OA. Osteoarthritis Cartilage 2010; 18: 563-571.

28. Koyama N, Okubo Y, Nakao K, Osawa K, Fujimura K, Bessho K. Pluripotency of mesenchymal cells derived from synovial fluid in patients with temporomandibular joint disorder. Life Sci 2011; 89: 741-747.

29. Sekiya I, Ojima M, Suzuki S, Yamaga M, Horie M, Koga H et al. Human mesenchymal stem cells in synovial fluid increase in the knee with degenerated cartilage and osteoarthritis. J Orthop Res 2012; 30: 943-949.

30. Hunter DJ, Gerstenfeld L, Bishop G, Davis AD, Mason ZD, Einhorn TA et al. Bone marrow lesions from osteoarthritis knees are characterized by sclerotic bone that is less well mineralized. Arthritis Res Ther 2009; 11: R11.

31. Goldring SR. Alterations in periarticular bone and cross talk between subchondral bone and articular cartilage in osteoarthritis. Ther Adv Musculoskelet Dis 2012; 4: 249-258.

32. Kusumbe AP, Ramasamy SK, Adams RH. Coupling of angiogenesis and osteogenesis by a specific vessel subtype in bone. Nature 2014; 507: 323-328.

33. Portal-Nunez S, Lozano D, Esbrit P. Role of angiogenesis on bone formation. Histol Histopathol 2012; 27: 559-566.

34. Kanczler JM, Oreffo RO. Osteogenesis and angiogenesis: the potential for engineering bone. Eur Cells Mater 2008; 15: 100-114. 
35. Nassiri SM, Rahbarghazi R. Interactions of mesenchymal stem cells with endothelial cells. Stem Cells Dev 2014; 23: 319-332.

36. Wang H, Yin Y, Li W, Zhao X, Yu Y, Zhu J et al. Over-expression of PDGFR-beta promotes PDGF-induced proliferation, migration, and angiogenesis of EPCs through PI3K/Akt signaling pathway. PLOS ONE 2012; 7: e30503.

37. Chien SY, Huang CY, Tsai CH, Wang SW, Lin YM, Tang CH. Interleukin-1beta induces fibroblast growth factor 2 expression and subsequently promotes endothelial progenitor cell angiogenesis in chondrocytes. Clin Sci 2016; 130: 667-681.

38. Honorati MC, Neri S, Cattini L, Facchini A. Interleukin-17, a regulator of angiogenic factor release by synovial fibroblasts. Osteoarthritis Cartilage 2006; 14: 345-352.

39. Kayakabe K, Kuroiwa T, Sakurai N, Ikeuchi H, Kadiombo AT, Sakairi T et al. Interleukin-6 promotes destabilized angiogenesis by modulating angiopoietin expression in rheumatoid arthritis. Rheumatology (Oxford) 2012; 51: 1571-1579.

40. Yang HL, Chang HC, Lin SW, Senthil Kumar KJ, Liao CH, Wang HM et al. Antrodia salmonea inhibits TNF-alpha-induced angiogenesis and atherogenesis in human endothelial cells through the down-regulation of NF-kappaB and up-regulation of Nrf2 signaling pathways J Ethnopharmacol 2014; 151: 394-406.

41. Hashimoto S, Matsumoto K, Gon Y, Maruoka S, Kujime K, Hayashi S et al. p38 MAP kinase regulates TNF alpha-, IL-1 alpha- and PAF-induced RANTES and GM-CSF production by human bronchial epithelial cells. Clin Exp Allergy 2000; 30: 48-55.

42. Hamilton JL, Nagao M, Levine BR, Chen D, Olsen BR, Im HJ. Targeting VEGF and its receptors for the treatment of osteoarthritis and associated pain. J Bone Miner Res 2016; 31: 911-924.
43. Maksymowych WP, Russell AS, Chiu P, Yan A, Jones N, Clare T et al. Targeting tumour necrosis factor alleviates signs and symptoms of inflammatory osteoarthritis of the knee. Arthritis Res Ther 2012; 14: R206.

44. Xu J, Wang Y, Li J, Zhang X, Geng Y, Huang Y et al. IL-12p40 impairs mesenchymal stem cell-mediated bone regeneration via CD4+ T cells. Cell Death Differ 2016; 23 . 1941-1951.

\section{(c) (1)}

Cell Death and Disease is an open-access journal published by Nature Publishing Group. This work is licensed under a Creative Commons Attribution 4.0 International License. The images or other third party material in this article are included in the article's Creative Commons license, unless indicated otherwise in the credit line; if the material is not included under the Creative Commons license, users will need to obtain permission from the license holder to reproduce the material. To view a copy of this license, visit http://creativecommons.org/licenses/by/4.0/

(C) The Author(s) 2017 